\title{
Analysis of the Epidemiological Characteristics of Rabies in Jingxi City, Guangxi from 2000 to 2020
}

\author{
Wang Fuchun ${ }^{1, ~ *, ~ L i a n g ~ G u o y u e ~}{ }^{1}$, Huang Yaming ${ }^{2,3}$ \\ ${ }^{1}$ Jingxi City Diseuse Control and Prevention, Jingxi, China \\ ${ }^{2}$ Guangxi Zhuang Automomous Region Center for Disease Prevention, Nanning, China \\ ${ }^{3}$ Basic Medical College of Medical University, Kunming, China
}

Email address:

jxcde@163.com (Wang Fuchun)

${ }^{*}$ Corresponding author

\section{To cite this article:}

Wang Fuchun, Liang Guoyue, Huang Yaming. Analysis of the Epidemiological Characteristics of Rabies in Jingxi City, Guangxi from 2000 to 2020. European Journal of Preventive Medicine. Vol. 9, No. 3, 2021, pp. 94-100. doi: 10.11648/j.ejpm.20210903.14

Received: May 25, 2021; Accepted: June 9, 2021; Published: June 21, 2021

\begin{abstract}
Objective Analyze the epidemiological characteristics of rabies in Jingxi City to provide a basis for formulating rabies control measures. Methods Descriptive epidemiological methods were used to analyze the surveillance data of rabies in Jingxi City from 2000 to 2020 . Results A total of 73 rabies cases and 73 cases of rabies were reported in Jingxi City from 2000 to 2020 , with an average annual incidence rate of $0.55 / 100,000$, and a case fatality rate of $100 \%$; cases were reported in 19 towns and townships; it occurred frequently in Summer and autumn, with $56.16 \%$ of cases from May to September. Mainly farmers, the incidence accounted for 84.93\%; male incidence rate was $0.70 / 100,000$, female incidence rate was $0.38 / 100,000$, male and female incidence ratio was 2.04:1; the main wonding animals were dogs, accounting for 95.89\%; humans No history of vaccination after exposure accounted for $97 \%$. Conclusion The rural area Jingxi City is a key area for rabies Prevention and control. It is necessary to strengthen the propaganda of rabies prevention knowledge, standardize post-exposure treatment, and carry out large-scale dog immunization. Comprehensive prevention measures such as immunization and hunting to effectively curb the epidemic of rabies. Background: Jingxi, Guangxi is located on the border of China and Vietnam. The border with Vietnam to the south is $152.5 \mathrm{~km}$ long. It has a subtropical monsoon climate. It has 19 villages and towns under its jurisdiction and has a total population of 670,000 . Among them, ethnic minority imports account for $99.40 \%$. The port was expanded to an international port with the approval of the state council, and the Yuexu port was also upgraded to a national first-level port. Border trade increased. Residents in rural areas have the habit of raising domestic dogs and eating dog meat. There are a large number of dogs, dog registration management, and immunization. The implementation of hunting and killing measures is not in place, the standardized disposal rate after exposure is low, and rabies epidemics occur from time to time.
\end{abstract}

Keywords: Rabies, Epidemic Characteristics, Analysis

\section{Introduction}

With the exception of Antarctica, there are reports of human rabies in all continents, except for Antarctica. Rabies has been eliminated in Western Europe, Canada, the United States, Japan, Malaysia and a few countries in Latin America. Australia has eliminated rabies in carnivorous animals. Rabies is still an important global public health Problem. There are about 60,000 rabies deaths every year. Among them, the number of rabies cases in developing countries accounts for $99 \%$. The epidemic is severe in Asia, Africa,
Latin America and the caribbean. The rabies epidemic in Asia ranks first in the world, and the number of rabies cases is estimated to be 30,000 per year. It is estimated that 20,000 to 30,000 people are affected each year, with an incidence rate of 2/100,000; Second to India, when the rabies epidemic peaked in 2007, the number of reported rabies cases was 3,300.

Rabies cases have been reported in all provinces across the country. From 1996 to 2008 , the top 10 provinces in the 
country for rabies epidemic were Guangxi, Hunan, Guizhou, Guangdong, Jiangxi, Jiangsu, Hubei, Henan, Sichuan and Anwei. The number of reported cases accounted for the total number of cases in the country $86.90 \%$. In 2007 , the national rabies epidemic peaked. After a series of rabies prevention and control measures were adopted, the national rabies epidemic continued to decline since 2008. In 2014, the number of reported rabies cases nationwide was less than 1,000 , which was a $72 \%$ decrease in the incidence rate from 2007.

Guangxi is a province with a high incidence of rabies in the country. After adopting comprehensive prevention measures such as "management, immunization, hunting and killing" of dogs, the rabies epidemic has been on a downward trend since 2013. A total of 912 rabies cases have been reported from 2011 to 2015 , compared with 2005 to 2010 , the cumulative number of reported 2009 cases decreased by $54.60 \%$.

Jingxi City entered the beginning of the 21 st century, and the rabies epidemic was a high incidence county in Guangxi. A total of 29 cases of rabies were reported from 2001 to 2002, of which 14 were in 2001 and 15 were in 2002. After adopting a series of rabies prevention and control measures, the rabies epidemic has dropped significantly after 2013. The number of reported rabies cases from 2016 to 2019 was 0 , and the number of reported rabies cases in 2020 was one. Rabies is still spreading.

Rabies is an animal-derived infectious disease caused by rabies virus (Rabies virus, RABV) infection. Rabies virus invades the human body mainly through damaged skin or mucous membranes. Sexual paralysis, also known as hydrophobia [1], is the only infectious disease in the world with a fatality rate of $100 \%[2,3]$. Rabies is a legally classified B infectious disease in my country. There is a habit of raising dogs and nursing homes in rural areas. The density of dogs is relatively high [4], but the management and immunity of dogs is poor. Rabies caused by dog bites is a relatively common fatality hurt. Jingxi City is an area with a high incidence of rabies. In order to understand the characteristics of rabies epidemic and provide a basis for formulating rabies prevention and control measures, the characteristics of rabies epidemic from 2000 to 2020 in Jingxi City are analyzed as follows.

\section{Materials and Methods}

\subsection{Data Source}

The rabies epidemic data comes from the "China Disease Surveillance Information Report Management System”, the rabies epidemic data, rabies case survey forms, and survey reports in Jingxi City from 2000 to 2020; the demographic data comes from JingXi Statistics Bureau.

\subsection{Case Diagnosis}

According to the National Ministry of Health's health industry standard WS281-2008 "Rabies Diagnostic standards", clinical diagnosis epidemiological investigation, monitoring and treatment of cases are carried out [5].

\subsection{Rabies Exposure Classification Criteria}

According to the Nation Ministry of Health "Rabies Post exposure Disposal Work Regulations (Trial)" (2006 version), the rabies exposure classification is determined: contact or feeding animals, or intact skin is licked as class 1 ; bare skin is lightly bitten, or there is on slight bleeding scratches and abrasions are grade 11; single or multiple penetrating skin bites or scratches, or broken skin is licked, or open wounds and mucous membranes are contaminated as grade 111 [6].

\subsection{Methods}

Descriptive epidemiological methods were used to analyzethe epidemic data by Excel 2003 software. The comparison of rates was performed by $\mathrm{x}^{2}$ test, and $\mathrm{P}<0.05$ was considered statistically significant.

\section{Results}

\subsection{Popular Profile}

From 2000 to 2020, a total of 73 cases of rabies were reported in Jingxi City, with an average annual incidence rate of $0.55 / 100,000$. The incidence of rabies in the city was between 0 and 2.59/100,000. With 2002 as the peak incidence, the incidence was $2.59 / 100,000$, followed by 2001 . With an incidence rate of 2.42/100,000, with an incidence rate of from 2016 to 2019, and an incidence in incidence in 2020, with 1 case of rabies reported. See picture 1 .

\subsection{Population Distribution}

Among the 73 cases of rabies, the youngest age is 5 years old and the oldest is 79 years old. The incidence is mainly concentrated in tho 50-75 years old age group. The 60 -year-old age group has the highest incidence rate of $2.70 / 100,000$, followed by the age group $\geq 75$ and 65 years old. The incidence rates were $2.15 / 100,000,1.91 / 100,000$; the incidence rates of $0-15$ years old, 20-45 years old, and over 50 years old Were $0.20 / 100,000$ (10/4783132), 0.35/100,000 (21/5898273), 1.69/100,000 (42/2484384), the incidence of different age groups has a statistically significant difference $\left(\mathrm{x}^{2}=72.32, \mathrm{P}<0.005\right)$, as shown in Figure 2. The male and female incidence ratio was 2.04: 1 , and the male and female incidence rates were $0.70 / 100,000 \quad(49 / 6976558)$ and $0.38 / 100,000$ (24/6189229), respectively. The difference in incidence between men and women was statistically significant $\left(x^{2}=9.10, P<0.01\right)$, see Table 1 . The occupational composition of the cases is dominated by farmers, accounting for $84.93 \%(62 / 73)$ of the total number of cases, followed by students, accounting for $9.59 \%$ (7/73) of the total number of cases, scattered children, retirees, workers, and migrants. 1 cases, respectively accounting for $1.37 \%$. 


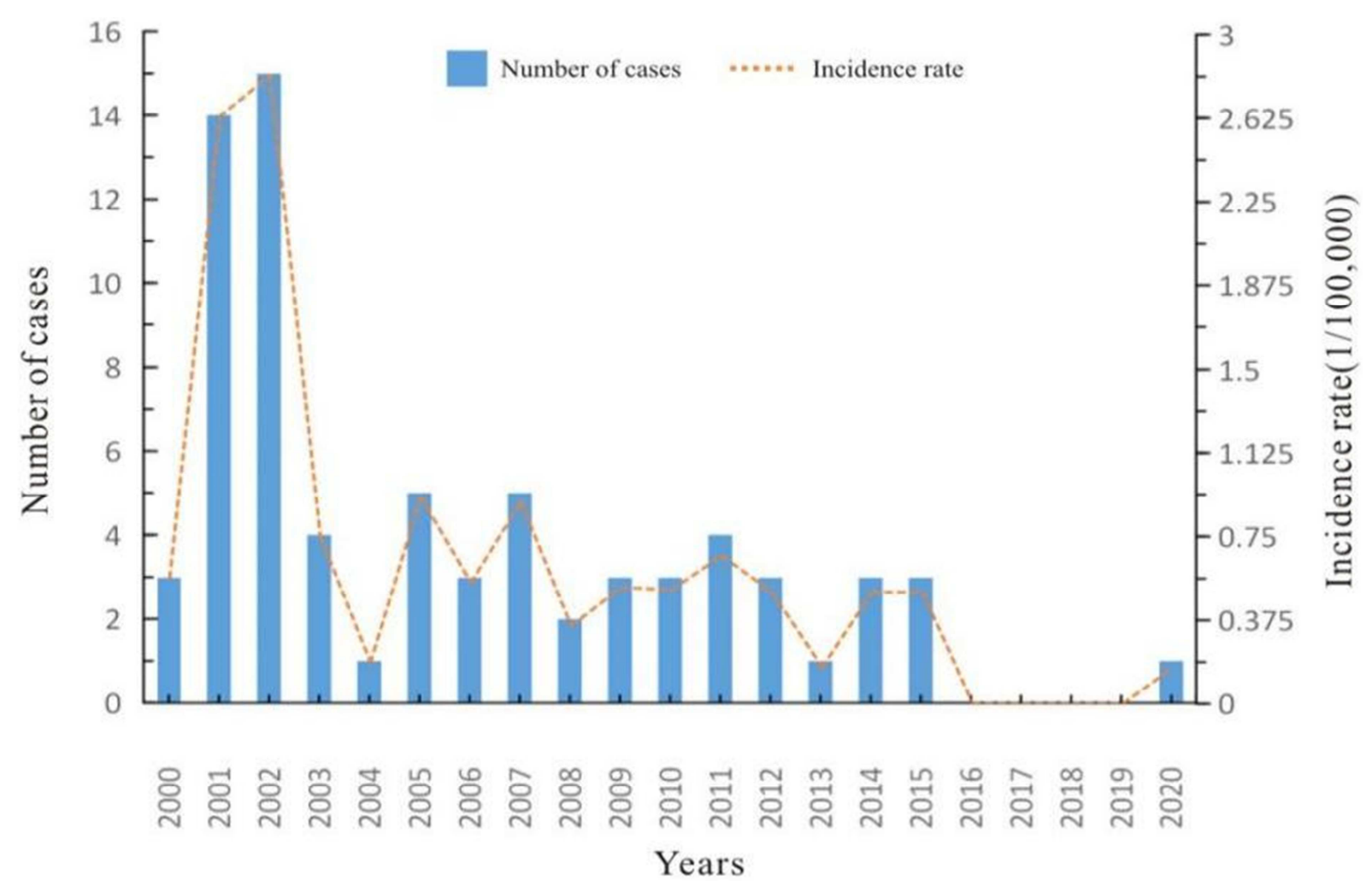

Figure 1. General situation of rabies epidemic in Jingx City from 2000 to 2020.

Table 1. Incidence of rabies in Jingxi City, Guangxi from 2000 to 2020.

\begin{tabular}{|c|c|c|c|c|c|c|c|c|c|c|c|}
\hline \multirow[b]{2}{*}{ Years } & \multirow{2}{*}{$\begin{array}{l}\text { Total } \\
\text { Population }\end{array}$} & \multirow{2}{*}{$\begin{array}{l}\text { Number } \\
\text { Of cases }\end{array}$} & \multirow{2}{*}{$\begin{array}{l}\text { Incidence } \\
\text { rate } \\
(1 / 100,000)\end{array}$} & \multirow{2}{*}{$\begin{array}{l}\text { Death } \\
\text { toll }\end{array}$} & \multirow{2}{*}{$\begin{array}{l}\text { Mortality } \\
\text { rate } \\
(\mathbf{1} / \mathbf{1 0 0 , 0 0 0 )})\end{array}$} & \multicolumn{3}{|l|}{ Male } & \multicolumn{3}{|l|}{ Female } \\
\hline & & & & & & $\begin{array}{l}\text { Total } \\
\text { Population }\end{array}$ & $\begin{array}{l}\text { Number } \\
\text { Of cases }\end{array}$ & $\begin{array}{l}\text { Incidence rate } \\
(1 / 100,000)\end{array}$ & $\begin{array}{l}\text { Total } \\
\text { Population }\end{array}$ & $\begin{array}{l}\text { Number } \\
\text { Of cases }\end{array}$ & $\begin{array}{l}\text { Incidence rate } \\
(\mathbf{1} / \mathbf{1 0 0 , 0 0 0 )}\end{array}$ \\
\hline 2000 & 572154 & 3 & 0.52 & 3 & 0.52 & 303184 & 2 & 0.66 & 268970 & 1 & 0.37 \\
\hline 2001 & 577411 & 14 & 2.42 & 14 & 2.42 & 305970 & 9 & 2.94 & 271441 & 5 & 1.84 \\
\hline 2002 & 578453 & 15 & 2.59 & 15 & 2.59 & 306522 & 11 & 3.59 & 271931 & 4 & 1.47 \\
\hline 2003 & 581100 & 4 & 0.69 & 4 & 0.69 & 307925 & 4 & 1.30 & 273175 & 0 & 0.00 \\
\hline 2004 & 585385 & 1 & 0.17 & 1 & 0.17 & 310196 & 1 & 0.32 & 275189 & 0 & 0.00 \\
\hline 2005 & 587788 & 5 & 0.85 & 5 & 0.85 & 311469 & 3 & 0.96 & 276319 & 2 & 0.72 \\
\hline 2006 & 594642 & 3 & 0.50 & 3 & 0.50 & 315101 & 2 & 0.63 & 279541 & 1 & 0.36 \\
\hline 2007 & 603153 & 5 & 0.83 & 5 & 0.83 & 319611 & 5 & 1.56 & 283542 & 0 & 0.00 \\
\hline 2008 & 615564 & 2 & 0.32 & 2 & 0.32 & 326187 & 0 & 0.00 & 289377 & 2 & 0.69 \\
\hline 2009 & 629793 & 3 & 0.48 & 3 & 0.48 & 333727 & 1 & 0.30 & 296066 & 2 & 0.68 \\
\hline 2010 & 644690 & 3 & 0.47 & 3 & 0.47 & 341621 & 2 & 0.59 & 303069 & 1 & 0.33 \\
\hline 2011 & 650689 & 4 & 0.61 & 4 & 0.61 & 344800 & 1 & 0.29 & 305889 & 3 & 0.98 \\
\hline 2012 & 652040 & 3 & 0.46 & 3 & 0.46 & 345516 & 2 & 0.58 & 306524 & 1 & 0.33 \\
\hline 2013 & 658351 & 1 & 0.15 & 1 & 0.15 & 348860 & 1 & 0.29 & 309491 & 0 & 0.00 \\
\hline 2014 & 658412 & 3 & 0.46 & 3 & 0.46 & 348893 & 2 & 0.57 & 309519 & 1 & 0.32 \\
\hline 2015 & 658676 & 3 & 0.46 & 3 & 0.46 & 349032 & 2 & 0.57 & 309644 & 1 & 0.32 \\
\hline 2016 & 658983 & 0 & 0.00 & 0 & 0.00 & 349195 & 0 & 0.00 & 309788 & 0 & 0.00 \\
\hline 2017 & 659103 & 0 & 0.00 & 0 & 0.00 & 349259 & 0 & 0.00 & 309844 & 0 & 0.00 \\
\hline 2018 & 662877 & 0 & 0.00 & 0 & 0.00 & 351259 & 0 & 0.00 & 311618 & 0 & 0.00 \\
\hline 2019 & 664258 & 0 & 0.00 & 0 & 0.00 & 351990 & 0 & 0.00 & 312268 & 0 & 0.00 \\
\hline 2020 & 672265 & 1 & 0.15 & 1 & 0.15 & 356233 & 1 & 0.28 & 316032 & 0 & 0.00 \\
\hline Total & 13165787 & 73 & 0.55 & 73 & 0.55 & 6976551 & 49 & 0.70 & 6189236 & 24 & 0.39 \\
\hline
\end{tabular}

\subsection{Time Distribution}

Cases of rabies in Jingxi City are reported all year round. The incidence of rabies is relatively stable from October to
February of the following year. The incidence starts to increase from March to April. The peak season is from May to September. The number of reported cases accounts for $56.16 \%$ (41/73), of the total number of cases. May and September are the two peaks of the disease. See Figure 3. 


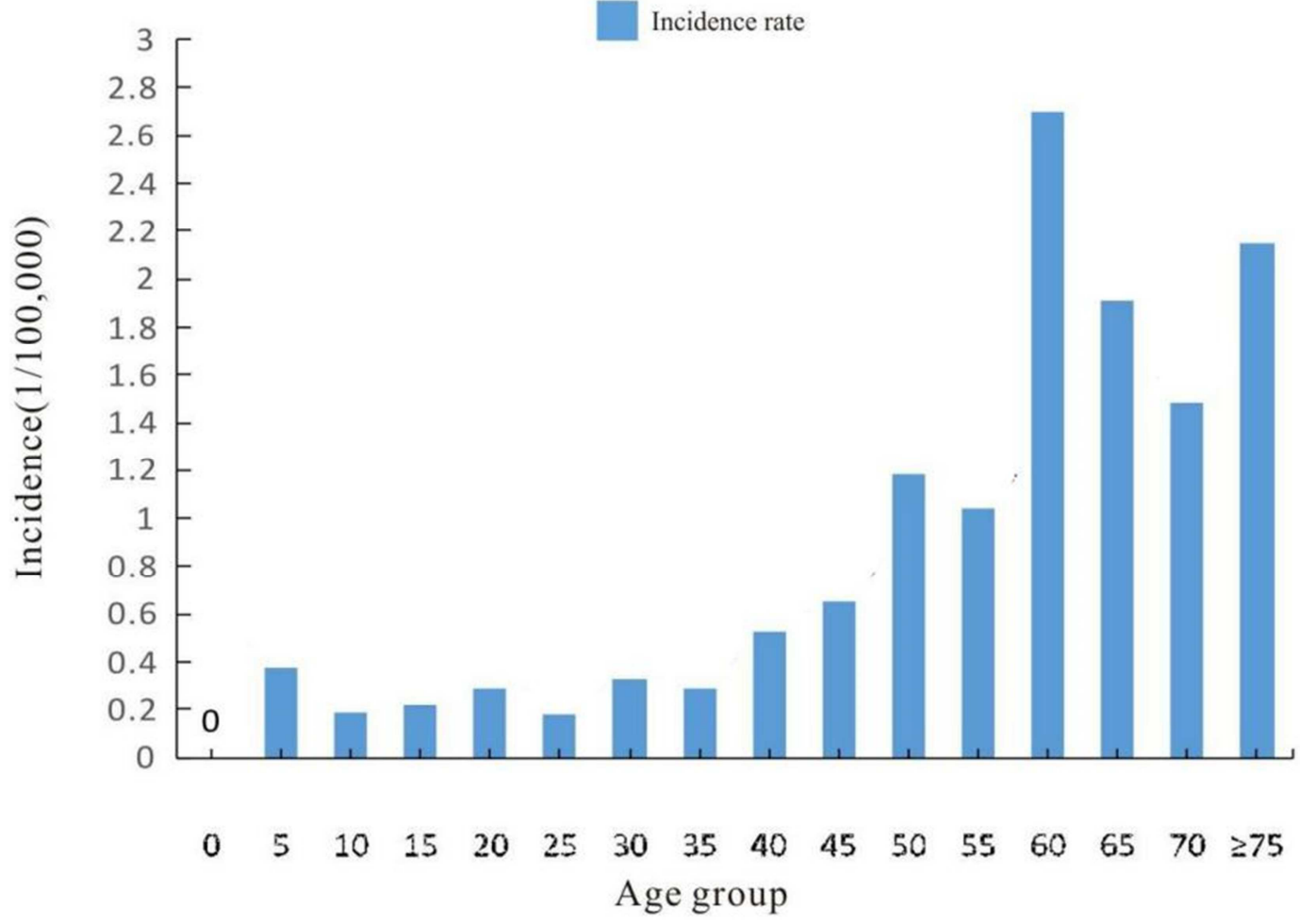

Figure 2. Distribution of Rabies Incidence by Age Group in Jingx City, Guangxi from 2000 to 2020.

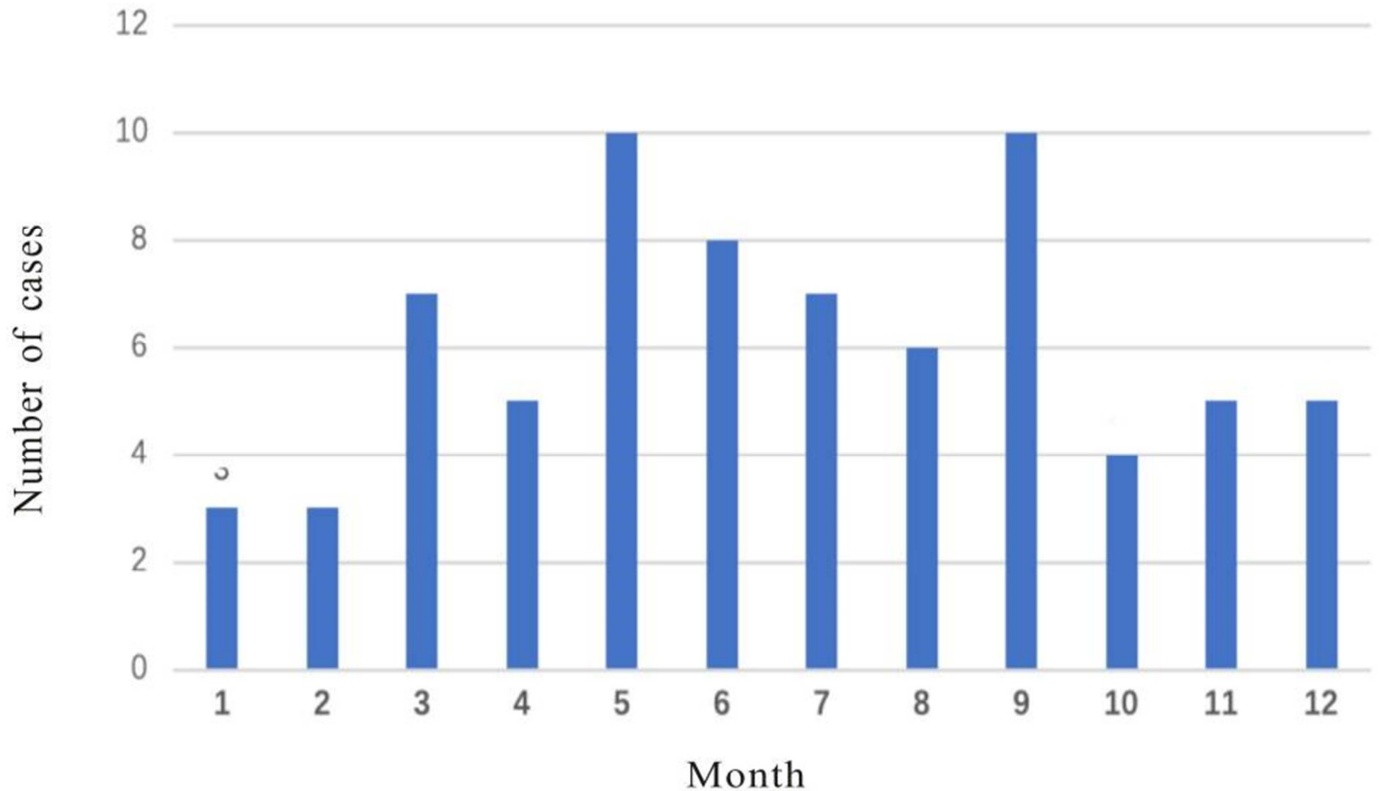

Figure 3. Monthly distribution of rabies cases in Jingx City, Guangxi from 2000 to 2020.

\subsection{Local Distribution}

Rabies cases were reported in 19 villages and towns in Jingxi City from 2000 to 2020. Longlin Township had the highest incidence rate of 1.70/100,000 (10/589134), followed by Renzhuang Township and Yuexu Township, with an incidence rate of 1.37/100,000 (4/291606), 1.36/100,000 $(3 / 220059)$. The lowest incidence rate is the floating population and GuoLe Township, the incidence is 0.03/100,000 (1/3909906), 0.22/100,000 (1/456771); from the distribution of urban and rural cases, the incidence rate in rural areas was $0.65 / 100,000(65 / 9874340)$, and the incidence rate in urban areas was $0.24 / 100,000$ (8/3291447). The difference in the distribution of urban and rural cases was statistically significant $\left(\mathrm{x}^{2}=7.68, \mathrm{P}<0.01\right)$, See Table 2 . 
Table 2. Regional distribution of rabies in Jingxi City, Guangxi from2000 to 2020.

\begin{tabular}{|c|c|c|c|c|c|c|c|}
\hline Township & $\begin{array}{l}\text { Average annual } \\
\text { Population }\end{array}$ & $\begin{array}{l}\text { Number of } \\
\text { cases }\end{array}$ & $\begin{array}{l}\text { Incidence rate } \\
(1 / 100,000)\end{array}$ & $\begin{array}{l}\text { Incidence } \\
\text { rank }\end{array}$ & Death toll & $\begin{array}{l}\text { Mortality rate } \\
(\mathbf{1} / \mathbf{1 0 0 , 0 0 0 )}\end{array}$ & Mortality rank \\
\hline Longlin & 589134 & 10 & 1.70 & 1 & 10 & 1.70 & 1 \\
\hline Renzhuang & 291606 & 4 & 1.37 & 2 & 4 & 1.37 & 2 \\
\hline Yuexu & 220059 & 3 & 1.36 & 3 & 3 & 1.36 & 3 \\
\hline Huadong & 310381 & 4 & 1.29 & 4 & 4 & 1.29 & 4 \\
\hline Wuping & 667086 & 8 & 1.20 & 5 & 8 & 1.20 & 5 \\
\hline Nanpo & 475272 & 5 & 1.05 & 6 & 5 & 1.05 & 6 \\
\hline Anning & 234864 & 2 & 0.85 & 8 & 2 & 0.85 & 8 \\
\hline Dizhou & 358890 & 3 & 0.84 & 9 & 3 & 0.84 & 9 \\
\hline Longbang & 287112 & 2 & 0.70 & 10 & 2 & 0.70 & 10 \\
\hline Tongde & 426301 & 3 & 0.70 & 10 & 3 & 0.70 & 10 \\
\hline Tunpan & 291795 & 2 & 0.69 & 11 & 2 & 0.69 & 11 \\
\hline Ande & 646653 & 4 & 0.62 & 12 & 4 & 0.62 & 12 \\
\hline Xingjing & 1358282 & 8 & 0.59 & 13 & 8 & 0.59 & 13 \\
\hline Xinjia & 608601 & 3 & 0.49 & 14 & 3 & 0.49 & 14 \\
\hline Hurun & 355951 & 1 & 0.28 & 16 & 1 & 0.28 & 16 \\
\hline Ludong & 733929 & 2 & 0.27 & 17 & 2 & 0.27 & 17 \\
\hline Guole & 456771 & 1 & 0.22 & 18 & 1 & 0.22 & 18 \\
\hline Floating Population & 3909906 & 1 & 0.03 & 19 & 1 & 0.03 & 19 \\
\hline Total & 13165787 & 73 & 0.55 & & 73 & 0.55 & \\
\hline
\end{tabular}

\subsection{Sources of Rabies}

The ways of feeding animals that bite people are $41.10 \%$ (30 animals) of their own dogs, 39.73\% (29 animals) of neighboring dogs, $6.85 \%$ (5 animals) of traded dogs, and $10.96 \%$ ( 8 animals) of foreign dogs. Own cats accounted for $1.37 \%$ (1 cat). Dog bites were exposed to dog bites, which accounted for $95.89 \%$ (70 cases), slaughtered dogs and processed dog meat products accounted for $2.74 \%$ ( 2 cases), and cat bites accounted for $1.37 \%$ ( 1 cases). The main cause of human bites was dog bites. Active attack injuries by dogs accounted for $58.90 \%$ (43 animals), self-defense injuries by dogs accounted for $30.14 \%$ (22 animals), and multible dog bites at the same time accounted for $8.22 \%$ (6 animals). Canine animals without immunization history accounted for $87.67 \%$ (64 animals), and unknown accounted for $12.33 \%$ (9 animals).

\subsection{Wound Exposure Treatment}

The most common dog bites the upper limbs and hands, accounting for $78.08 \%$ (57 cases), the lower limbs and feet accounted for $8.22 \%$ ( 6 cases), the head and face accounted for $13.70 \%$ (10 cases), of which multi-site bites accounted for $6.85 \%$ ( 5 cases); Wound exposure classification grade I, grade II, grade III accounted for $4.11 \%$ ( 3 cases), $39.73 \%$ ( 29 cases), $56.16 \%$ (41 cases) respectively. After wound exposure, patients without treatment accounted for $93.15 \%$ (68 cases), simple self-treatment accounted for $2.74 \%$ ( 2 cases), and doctors treated $4.11 \%$ ( 3 cases). Among the 73 rabies patients, $91.78 \%$ (67 cases) had no history of rabies vaccination, $8.22 \%$ (6 cases) had a history of vaccination, and among the 6 cases with a history of vaccination, 4 people became ill after the third shot. Two people became ill after the fourth shot; $9.76 \%$ (4/41) were vaccinated for those exposed to grade III wounds, and $6.90 \%(2 / 29)$ were vaccinated for those exposed to grade
II wounds. Among the 6 people who were vaccinated after wound exposure, no anti-rabies immunoglobulin or anti-rabies serum was used.

\section{Clinical Manifestations}

The clinical manifestations of 73 rabies patients wer manic, with a rapid onset of $93.15 \%$ (68 cases) and a slow onset of $6.85 \%$ ( 5 cases).

\subsection{Incubation Period}

The shortest incubation period of patients is 20 days and the longest is 4 years; the incubation period is $0-3$ months accounting for $61.64 \%$ (45 cases), of which 1 month is the most accounting for $34.25 \%$ ( 25 cases), followed by $<1$ month accounting for $12.33 \%$ ( 9 cases); $4-11$ months accounted for $23.29 \%$ (17 cases), $1-3$ years accounted for $12.33 \%$ (9 cases), $\geq 4$ years accounted for $2.74 \%$ ( 2 cases).

\subsection{Prodromal Period}

Among 73 patients, the clinical manifestations were fatigue accounted for $63.01 \%$ (46 cases), headache accounted for $43.84 \%$ ( 32 cases). Fever $37.5-40^{\circ} \mathrm{C}$ accounted for $18.25 \%$ (7 cases).

\subsection{Excitement Period}

Among the 73 cases of rabies, the clinical manifestations wer fear $100 \%$ ( 73 cases), $93.15 \%$ fear of water (68 cases), $97.26 \%$ fear of wind (71 cases), 91.78\% fear of light (67 cases). fear of sound Accounted for $80.82 \%$ (59 cases), excitement accounted for $71.23 \%$ ( 52 cases), mania accounted for $41.10 \%$ (30 cases), convulsions accounted for $34.25 \%$ ( 25 cases), coma accounted for $9.59 \%$ ( 7 cases), death accounted for $100 \%$ ( 73 cases). 


\subsection{Patient's Course}

Among the 73 rabies patients, the shortest time from onset to death was 1 day, the longest was 8 days, and 2-5 days accounted for $90.41 \%$ (66 cases). Death was caused by pharyngeal muscle spasm and suffocation or respiratory and circulatory failure [7].

\section{Discussion}

Jingxi City is an area with a high prevalence of rabies in Baise City, Guangxi. Since rabies cases were reported in 1984, a total of 58 rabies cases have been reported from 1984 to 1990 , with a case fatality rate of $100 \%$, with 16 cases in 1984 being the most, followed by 1986 year of 15 rabies cases. After 1990, rabies was effectively controlled for a time. the is basically consistent with the reports from all parts of the country and Guangxi $[8,9]$. Rabies has been on the rise since 2000. The incidence rates in 2001 and 2002 were $2.42 / 100,000$ (14/577411) and 2.59/100,000 (15/578453) respectively. Through active Prevention and control measures, extensive health education has been carried out to strengthen Dog management, immunization, hunting and killing, and timely treatment and treatment measures after exposure to human and dog injuries, the incidence of rabies dropped to 0 from 2016 to 2019 , but the incidence of rabies began to rise in 2020 , and 1 case of rabies was reported in 2020. It shows that although the rabies prevention and control work in Jingxi City has achieved certain results, there is still a big gap between the goal of eliminating rabies.

The age composition of rabies in Jingxi City is mainly concentrated in tho 50-75 year-old age group (57.53\%). primary school students and scattered children also have the disease, mainly farmers in rural areas $(84.93 \%)$. The male to female ratio is 2.04: 1. Population distribution The characteristics are mostly men, farmers, and people over the age of 50, which is basically consistent with the situation in all provinces across the country $[10,11]$. The main reason may be the lack of knowledge about rabies among the elderly, elementary school students and scattered children, and they cannot be treated and treated in time after exposure; primary school students and scattered children lack the awareness of danger recognition and self-protection ability due to their young age, and fail to inform their parents in time after exposure to rabies. Wound exposure The lack of timely disposal is related to vaccine immunization. At the same time, most of the parents of rural elementary school students and scattered children go out to work for many years, and there are only left-behind elderly and children at home, which is related to the lack of awareness of the serious harm of rabies. There are more men with rabies than women. This may be related to the aggressiveness of men, who are more likely to be exposed to dog injuries such as fighting animals and playing games. At the same time, men do not pay enough attention to health and are more likely to have fluke psychology.

There are more rabies in Jingxi City in rural areas than in urban areas. It may be related to the high density of dogs in rural areas and the low vaccination rate [12]. Farmers in rural areas or left-behind elderly groups have the habit of keeping dogs in nursing homes and fong of dog meat. There are more dogs in the family and lack of vaccination management for dogs. There are many exposure opportunities for dog injuries such as feeding; the economic and social development and medical and health conditions in rural areas are relatively poor, health and disease prevention publicity and education are weak, and the self-protection awareness of the population is weak. After exposure to dog injuries, most of the dogs have not been exposed to post-exposure treatment, vaccination. Injection of anti-rabies immunoglobulin or anti-rabies serum is related $[13,14]$.

The seasonal distribution of rabies in Jingxi City is mostly in summer and autumn, and relatively less in winter and spring, which may be related to the hot temperatures in summer and autumn. People go out freguently, wear thin clothes, and increase exposure ti dog injuries. They are more likely to be bitten by dogs [15]; on the other hand, it may be related to the hot weather, and canine animals are irritable and easy to be irritated and attacked [16]. The regional distribution of rabies in Jingxi City ranks among the top 5 in the cumulative number of reported cases in Longlin Town, Renzhuang Township, Yuexu Town, Huadong Town, and Wuping Town, accounting for $39.73 \%$ of the total number of cases. It may ba related to the high density of local imports and the increase in the number of dogs raised, which increases the risk of dog bite exposure; as the cases are distributed in different towns and rural areas, it has caused a sporadic epidemic trend.

Post-exposure treatment of rabies is the only effective way to prevent rabies after exposure. WHO believes that timely treatment of wounds, standardized use of vaccines, and use of rabies immunoglobulin when necessary are effective in preventing rabies deaths by $100.00 \%$ [17]. However, the results of this survey showed that $93.15 \%$ of the cases did not have any treatment on the wound after exposure, $91.78 \%$ of the cases were not vaccinated, and $100 \%$ of the cases were not injected with anti-rabies immunoglobulin or anti-rabies serum for passive immunization. The main reason for the high incidence of rabies. The reasons for the lack of immune management of canine animals and the low disposal rate after human exposure may be due to the lack of awareness of the hazards of rabies among the urban and rural residents of the city, the lack of corresponding knowledge of rabies prevention and treatment and the knowledge of standardized treatment after exposure. The illness consciousness is weak, and the dog failed to the treated in time after being injured.

WHO held the "International Conference on Rabies Elimination" in Geneva on December 10-11, 2015, hoping to strengthen rabies prevention work on a global scale, and ultimately eliminate rabies by 2030 . The meeting pointed out that there are currently two main ways to prevent rabies, one is to vaccinate people quickly after exposure; the other is to vaccinate dogs to curb the spread of rabies virus from the source [18]. Rabies is a animal-borne infectious disease with a fatality rate of $100 \%$. Therefore, health education should be strengthened, high-risk populations should be more aware of 
the serious hazards of rabies, and self-protection awareness and ability should be improved; agricultural, animal husbandry and veterinary departments should strengthen dog immunization, control the spread of rabies at the source, and adhere to dog registration management and immunization systems; The health department should strengthen the prevention and treatment of human rabies after exposure, treat the wounds in a timely and correct manner, and rationally apply rabies vaccines and passive rabies immunization preparations. Speed up the process of rabies prevention and control, and achieve the goal of effective rabies control.

\section{Conclusion}

The rural area of Jingxi City is a key area for rabies prevention and control. There are a large number of dogs in rural areas, but management measures are lagging, and preventive measures after dog injuries are improper, rural residents have insufficient awareness of the serious hazards of rabies and the importance of vaccination, and standardized treatment rates after exposure Low levels are the main reason for the increased incidence of rabies. Rabies prevention knowledge propaganda in rural areas, standardized treatment after exposure, and large-scale immunization of dogs should be increased. The government should lead the government and all relevant functional departments should cooperate closely to conscientiously implement the comprehensive "management, immunization, and killing" of dogs. preventive measures to effectively curb the epidemic of rabies.

\section{References}

[1] WHO. Expert Consultation on rabies: Second Report, Geneva: World Health Organization, 2013.

[2] The Ministry of Health of the People's Republic of China, the status of rabies control in China, Beijing: People's Medical Publishing House, 2009: 1-12.

[3] Wang Fuchun (2010). Current research status of rabies epidemic and prevention in my country, Medical Animal Control, 26 (7): 611-613.

[4] Zha Risheng, YiBo, Tang xuefeng, et al (2010). Investigation on Dog Raising and Resident Dog Injury Treatment in Rural Areas of Yuyao City, Chian Rural Health Service Management, 30 (7): 562-564.

[5] Standards of the Ministry of Health of the People's Republic of
Chian, WS 281-2008 Rabies Diagnostic Standards, Beijing: People's Medical Publishing House, 2008.

[6] Chinese Center for Disease Control and Prevention, Rabies Prevention and Control Guidelines (2016 Edition), [EB/OL]. http://www.chinacdc.cn/zxdt/201304/120130402-79381.Htm.

[7] Shi Yinghong, Liu Changdi, Long Bo (2011). Clinical analysis of 29 Cases of rabies, Chinese Journal of Zoonoses, 27 (2): 165-166.

[8] Editor-in-Chief Wang Guoqiang, 60 years of disease prevention and control in china, Beijing: China Population press, 2015, pp. 169-173.

[9] Qin Zhongying, Deng Xingchao, Kong Qinglin, et all (2008). Epidemiological analysis of rabies in Guigang City from 1996-2006, Applied Preventive, 14 (1): 36-38.

[10] Qu Zhenning, Chen Qiaoge, Wang Tingting, et al (2015). Analysis of the epidemiological characteristics of rabies in Henan Province from 2005 to 2013, Modern Preventive Medicine, 42 (14): 2052-2054.

[11] Wu Hui, Song Sen, Shen Qinxin, et al (2011). Epidemiological analysis of rabies in China from 1996 to 2009, Disease Surveillance, 26 (6): 427-430.

[12] Yu Feng, Zhang Liping, Jin Baofang, et al (2014). The results of rabies control in Minhang District, Shanghai from 1993 to 2013, Chinese Journal of Disease Control, 18 (10): 926-930.

[13] Liu Gang, Lu Ziyan, Shan Fuxiang, et al (2013). Analysis of the epidemiological characteristics of rabies-exposed populations in Shenzhen from 2005 to 2011, Medical Animal control, 29 (5): 514-516.

[14] Zhuang Yan, Yu Chun, Hu Jing, et al (2013). Analysis of the epidemic status of rabies in Guizhou Province from 2001 to 2010 and discussion of its Prevention and control measures, Medical Animal Control, 29 (1): 1214-1216.

[15] Chen Weihua, Liu Xiaofeng He Qingyi, el al (2010). Analysis of the epidemiological characteristics and epidemic factors of rabies from 1990-2009 in Chenzhou City, Hunan Province, Medical Animal Control, 26 (10): 900-903.

[16] Lin Yun, Li Yuefeng, Zhou Wanling (2013). Analysis of the epidemiological characteristics of rabies in Jiaxing City, Zhejiang Province from 1983 to 2011, Chinese Journal of Vector Biology and Control, 24 (1): 72-74.

[17] Wang Dong, Wu Jiabing, Shi Guoqing (2012). Analysis of the population and host animals exposed to rabies at rural surveillance sites in Anhui Province from 2005 to 2012, Disease Surveillance, 27 (3): 191-194.

[18] Yang Shengzhi. The World Health Organization hopes to make rabies history in 2030, Medical Reference News/2015/December/17/G08 edition. 